\title{
Absolute ruin problems in a compound Poisson risk model with constant dividend barrier and liquid reserves
}

\author{
Dan Peng ${ }^{1 *}$, Donghai Liu' and Zhenting Hou²
}

\section{"Correspondence:}

danpengdanpeng@126.com 'School of Mathematics and Computational Science, Hunan University of Science and Technology, Xiangtan, Hunan 411201, P.R. China Full list of author information is available at the end of the article

\section{第 Springer}

\begin{abstract}
In this paper, we consider a compound Poisson surplus model with constant dividend barrier and liquid reserves under absolute ruin. When the surplus is negative, the insurer is allowed to borrow money at a debit interest rate to continue the business; when the surplus is below a fixed level $\Delta$, the surplus is kept as liquid reserves, which do not earn interest; when the surplus attains the level $\Delta$, the excess of the surplus over the level receives interest at a constant rate; when the surplus reaches a higher level $b$, the excess of the surplus above $b$ is all paid out as dividends to shareholders of the insurer. We first derive the integro-differential equations satisfied by the moment-generating function and moment of the discounted dividend payments until absolute ruin. Then, applying these results, we get explicit expressions of them for exponential claims and discuss the impact of the model parameters on the expected dividend payments by numerical examples.
\end{abstract}

MSC: $91 \mathrm{~B} 30$

Keywords: absolute ruin; dividend payments; liquid reserve; moment-generating function; interest

\section{Introduction}

In the classical compound Poisson surplus model, $U(t)$ is given by

$$
U(t)=u+c t-S(t)=u+c t-\sum_{i=1}^{N(t)} X_{i}, \quad t \geq 0
$$

where $U(0)=u$ is the initial surplus, $c>0$ is the premium rate, $\{N(t), t \geq 0\}$ is a Poisson process with intensity $\lambda>0$, which denotes the claim numbers in the interval $[0, t]$, and $\left\{X_{i}, i \geq 1\right\}$ (representing the sizes of claims and independent of $\{N(t), t \geq 0\}$ ) is a sequence of independent and identically distributed nonnegative random variables with common distribution function $F(x)=1-\bar{F}(x)$, which satisfies $F(0)=0$ and has a mean $\mu=\int_{0}^{\infty} \bar{F}(x) d x>0$.

In the recent study of risk theory, the classical compound Poisson surplus model has been modified to adopt economic and financial factors such as interest and dividends. The feature of debit interest assumes that the insurer is allowed to borrow money at a

(c) 2016 Peng et al. This article is distributed under the terms of the Creative Commons Attribution 4.0 International License (http://creativecommons.org/licenses/by/4.0/), which permits unrestricted use, distribution, and reproduction in any medium, provided you give appropriate credit to the original author(s) and the source, provide a link to the Creative Commons license, and indicate if changes were made. 
debit interest rate $\beta>0$ to pay claims when the surplus turns negative. As the insurer pays the debts from its premium income, the negative surplus may return to a positive level. When the premium income is not enough to pay the debit interest (that is, the surplus falls below $-\frac{c}{\beta}$ ), the absolute ruin is said to occur. In recent years, the issue of absolute ruin has received considerable attentions in the actuarial literature. See, for example, Embrechts and Schmidli [1], Cai [2], Yuen et al. [3], Wang et al. [4], and Yin and Wang [5]. For example, Yin and Wang [5] study absolute ruin questions for the perturbed compound Poisson risk process with investment and debit interests by the expected discounted penalty function at absolute ruin. On the other hand, even if an insurer invests all his positive surplus into a risk-free asset, in certain condition, only the excess of the surplus over a certain level can receive interest. To adopt a more flexible and tractable model, Embrechts and Schmidli [1] investigated the absolute ruin probability for a more complicated risk model. They assumed that the company can borrow money when the surplus is negative and receive interest for capital above a certain level. Furthermore, Cai et al. [6] considered the following special model of Embrechts and Schmidli [1]:

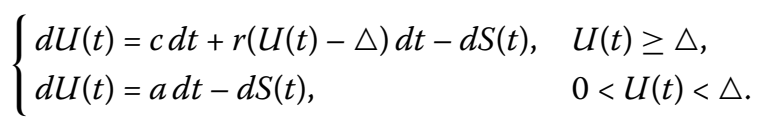

In (1.1), an insurer's surplus is below a certain level $\Delta>0$ and is kept as liquid reserves. As the surplus attains the level $\Delta$, the excess of the surplus above $\Delta$ will earn interest at a constant interest force $r>0$. They studied the Gerber-Shiu function and discussed the impact of interest and liquid reserves on the ruin probability.

On the other hand, the surplus of the insurer with a certain dividend strategy has also been receiving more and more attention, including [1, 7-9]. For instance, de Finetti [10] studied the dividend strategy in a discrete process. Lin et al. [9] investigated the classical risk model with constant dividend barrier and analyzed the Gerber-Shiu discounted penalty function at ruin. Albrecher et al. [11] considered the distribution of dividend payments in the Sparre Andersen model with constant dividend barrier. Cai et al. [12] considered a more general model that incorporates the notion of threshold strategy. Based on the model (1.1), they assume that if the surplus continues to surpass a higher level $b \geq \Delta$, then the excess of the surplus above $b$ is paid out as dividends to the insurer's shareholders at a constant dividend rate, and no interest is earned on the surplus over the threshold level $b$, and they discuss the interactions of the liquid reserve level, the interest rate, and the threshold level in the proposed risk model by studying the expected discounted penalty function and the expected present value of dividends paid up to the time of ruin. More specifically, they assume that the portion of the surplus is below a present level $\Delta$ is liquid, and the amount in excess of this level is invested under a deterministic interest rate. Instead of implementing a threshold in Cai et al. [12], Sendova and Zhang [13] consider a percentage of the current surplus of the insurer and also study the expected discounted penalty function at ruin.

Motivated by these works, based on the model (1.1), we consider a more general model that incorporates the dividend strategy and debit interest. We assume that if the surplus is negative, then the insurer can borrow money at a debit interest $\beta>r$. If the surplus surpass a higher level $b \geq \triangle$, then the excess of the surplus above $b$ is paid out as dividends to the 
shareholders at a constant dividend rate $c+r(b-\triangle)$. The resulting surplus process $U_{b}(t)$ can be described by

$$
d U_{b}(t)= \begin{cases}-d S(t), & U_{b}(t)>b, \\ \left(c+r\left(U_{b}(t)-\Delta\right)\right) d t-d S(t), & \Delta \leq U_{b}(t) \leq b, \\ c d t-d S(t), & 0 \leq U_{b}(t)<\Delta, \\ \left(c+\beta U_{b}(t)\right) d t-d S(t), & -\frac{c}{\beta}<U_{b}(t)<0,\end{cases}
$$

where $U_{b}(0)=u$ is the initial surplus, $b$ is the constant level of dividend barrier, $\beta$ is the debit interest rate, $r$ is the credit interest, $c$ is the premium rate, and $S(t)=\sum_{i=1}^{N(t)} X_{i}$ is the aggregate Poisson claim-amount process.

Define $T_{u}^{b}=\inf \left\{t: U_{b}(t) \leq-\frac{c}{\beta}\right\}$ as the time of absolute ruin $\left(T_{u}^{b}=\infty\right.$ if $U_{b}(t)>-\frac{c}{\beta}$ for all $t>0)$. Let $D(t)$ be the cumulative amount of dividends up to time $t$, and $\alpha>0$ be the force of interest. Then

$$
D_{u, b}=\int_{0}^{T_{u}^{b}} e^{-\alpha t} d D(t)
$$

is the present value of all dividends until $T_{u}^{b}$.

In the sequel, we consider the moment-generating function

$$
M(u, y, b)=E\left[e^{y D_{u, b}}\right], \quad-\frac{c}{\beta}<u \leq b,
$$

where $y$ is such that $M(u, y, b)$ exists. We denote the $n$th moment of the discounted dividends by

$$
V_{n}(u, b)=E\left[D_{u, b}^{n}\right], \quad-\frac{c}{\beta}<u \leq b, n \in N .
$$

Note that $V_{0}(u, b) \equiv 1$ and, when $n=1, V_{1}(u, b)=V(u, b)$ is the expectation of $D_{u, b}$. We will always assume that $M(u, y, b)$ and $V_{n}(u, b)$ are sufficiently smooth functions in $u$ and $y$, respectively.

The rest of the paper is organized as follows. In Section 2, we get the integro-differential equations for the moment-generating function and the $n$th moment of the discounted dividends. In Section 3, we find their explicit expressions for exponential claims and discuss the impact of the model parameters on the expected dividend payments by numerical examples.

\section{Integro-differential equations}

In this section, we study the moment-generating function $M(u, y, b)$, which has been discussed in various surplus processes; for example, see Albrecher et al. [11], Cheung (2008) etc. Similarly, we can analyze the moments of $D(u, b)$ through $M(u, y, b)$; since $M(u, y, b)$ has different paths for $-\frac{c}{\beta}<u \leq b$, we define

$$
M(u, y, b)= \begin{cases}M_{1}(u, y, b), & -\frac{c}{\beta}<u<0 \\ M_{2}(u, y, b), & 0 \leq u<\Delta \\ M_{3}(u, y, b), & \Delta \leq u \leq b\end{cases}
$$


Theorem 2.1 $M_{1}(u, y, b), M_{2}(u, y, b)$, and $M_{3}(u, y, b)$ satisfy the following system of integrodifferential equations:

$$
\begin{aligned}
& (\beta u+c) \frac{\partial M_{1}}{\partial u}(u, y, b)-\alpha y \frac{\partial M_{1}}{\partial y}(u, y, b)-\lambda M_{1}(u, y, b)+\lambda \bar{F}\left(u+\frac{c}{\beta}\right) \\
& \quad+\lambda \int_{0}^{u+\frac{c}{\beta}} M_{1}(u-x, y, b) d F(x)=0, \quad-\frac{c}{\beta}<u<0, \\
& c \frac{\partial M_{2}}{\partial u}(u, y, b)-\alpha y \frac{\partial M_{2}}{\partial y}(u, y, b)-\lambda M_{2}(u, y, b)+\lambda \int_{0}^{u} M_{2}(u-x, y, b) d F(x) \\
& \quad+\lambda \int_{u}^{u+\frac{c}{\beta}} M_{1}(u-x, y, b) d F(x)+\lambda \bar{F}\left(u+\frac{c}{\beta}\right)=0, \quad 0 \leq u<\Delta, \\
& {[r(u-\Delta)+c] \frac{\partial M_{3}}{\partial u}(u, y, b)-\alpha y \frac{\partial M_{3}}{\partial y}(u, y, b)-\lambda M_{3}(u, y, b)} \\
& \quad+\lambda \int_{0}^{u-\Delta} M_{3}(u-x, y, b) d F(x)+\lambda \int_{u-\Delta}^{u} M_{2}(u-x, y, b) d F(x) \\
& \quad+\lambda \int_{u}^{u+\frac{c}{\beta}} M_{1}(u-x, y, b) d F(x)+\lambda \bar{F}\left(u+\frac{c}{\beta}\right)=0, \quad \Delta \leq u \leq b,
\end{aligned}
$$

with boundary conditions:

$$
\begin{aligned}
& M_{1}\left(-\frac{c}{\beta}, y, b\right)=1, \\
& \left.\frac{\partial M_{3}(u, y, b)}{\partial u}\right|_{u=b}=y M_{3}(b, y, b), \\
& M_{1}(0-, y, b)=M_{2}(0, y, b), \quad M_{2}(\Delta-, y, b)=M_{3}(\triangle, y, b), \\
& \left.\left(c \frac{\partial M_{1}(u, y, b)}{\partial u}-\alpha y \frac{\partial M_{1}(u, y, b)}{\partial y}\right)\right|_{u=0-} \\
& \quad=\left.\left(c \frac{\partial M_{2}(u, y, b)}{\partial u}-\alpha y \frac{\partial M_{2}(u, y, b)}{\partial y}\right)\right|_{u=0}, \\
& \left.\left(c \frac{\partial M_{2}(u, y, b)}{\partial u}-\alpha y \frac{\partial M_{2}(u, y, b)}{\partial y}\right)\right|_{u=\Delta-} \\
& \quad=\left.\left(c \frac{\partial M_{3}(u, y, b)}{\partial u}-\alpha y \frac{\partial M_{2}(u, y, b)}{\partial y}\right)\right|_{u=\Delta} .
\end{aligned}
$$

Proof When $-\frac{c}{\beta}<u<0$, we consider the infinitesimal time from 0 to $t$, and three distinct events can happen: no claim in $(0, t)$, a claim in $(0, t)$ without occurring ruin, a claim in $(0, t)$ with occurring ruin. Conditioning on the time and amount of the first claim, we obtain that

$$
\begin{aligned}
M_{1}(u, y, b)= & (1-\lambda t) M_{1}\left(u e^{\beta t}+c \frac{e^{\beta t}-1}{\beta}, y e^{-\alpha t}, b\right)+\lambda t \int_{u e^{\beta t}+c \frac{e^{\beta t}-1}{\beta}+\frac{c}{\beta}}^{\infty} d F(x) \\
& +\lambda t \int_{0}^{u e^{\beta t}+c \frac{e^{\beta t}-1}{\beta}+\frac{c}{\beta}} M_{1}\left(u e^{\beta t}+c \frac{e^{\beta t}-1}{\beta}-x, y e^{-\alpha t}, b\right) d F(x)+o(t) .
\end{aligned}
$$


Letting $h_{\beta}(t, u)=u e^{\beta t}+c^{e^{\beta t}-1} \frac{\beta}{\beta}-u=(c+\beta u) \frac{e^{\beta t}-1}{\beta}$, we observe that $h_{\beta}(t, u) \rightarrow 0$ as $t \rightarrow 0$. By Taylor expansion we have

$$
\begin{aligned}
& M_{1}\left(u e^{\beta t}+c \frac{e^{\beta t}-1}{\beta}, y e^{-\alpha t}, b\right) \\
& \quad=M_{1}(u, y, b)+(\beta u+c) t \frac{\partial M_{1}(u, y, b)}{\partial u}-\alpha y t \frac{\partial M_{1}(u, y, b)}{\partial y}+o(t) .
\end{aligned}
$$

Substituting this expression into (2.8), dividing both sides of (2.8) by $t$, and letting $t \rightarrow 0$, we get (2.1). Similarly, we obtain (2.2) and (2.3).

When $u=-\frac{c}{\beta}$, absolute ruin is immediate, namely, no dividend is paid, and we obtain (2.4).

When $u=b$,

$$
\begin{aligned}
M_{3}(b, y, b)= & (1-\lambda t) e^{y[c+r(b-\Delta)] t} M_{3}\left(b, y e^{-\alpha t}, b\right) \\
& +\lambda t \int_{0}^{b-\Delta} M_{3}\left(b-x, y e^{-\alpha t}, b\right) d F(x) \\
& +\lambda t \int_{b-\Delta}^{b} M_{2}\left(b-x, y e^{-\alpha t}, b\right) d F(x) \\
& +\lambda t \int_{b}^{b+\frac{c}{\beta}} M_{1}\left(b-x, y e^{-\alpha t}, b\right) d F(x) \\
& +\lambda t \int_{b+\frac{c}{\beta}}^{\infty} d F(x)+o(t) .
\end{aligned}
$$

By similar methods we obtain the following equation from (2.9):

$$
\begin{aligned}
\alpha y \frac{\partial M_{3}(u, y, b)}{\partial y}= & {[y(r(b-\triangle)+c)-\lambda] M_{3}(b, y, b)+\lambda \bar{F}\left(b+\frac{c}{\beta}\right) } \\
& +\lambda \int_{0}^{b-\Delta} M_{3}(b-x, y, b) d F(x)+\lambda \int_{b-\Delta}^{b} M_{2}(b-x, y, b) d F(x) \\
& +\lambda \int_{b}^{b+\frac{c}{\beta}} M_{1}(b-x, y, b) d F(x) .
\end{aligned}
$$

Letting $u \uparrow b$ in (2.3) and comparing it with (2.10), we obtain (2.5).

Next, we prove condition (2.6). Here we only prove $M_{2}(\Delta-, y, b)=M_{3}(\Delta, y, b)$. For $0 \leq$ $u<\Delta$, let $\tau_{\Delta}$ be the time that the surplus reaches $\Delta$ for the first time from $0 \leq u<\Delta$. As before, we know that $t_{1}$ is the time that the surplus reaches $\Delta$ for the first time from $0 \leq u<\Delta$ with no claims. Then, by the Markov property of $U_{b}(t)$,

$$
\begin{aligned}
M_{2}(u, y, b) & =E\left[I\left(\tau_{\Delta}<T_{u}^{b}\right) e^{y D_{u, b}}\right]+E\left[I\left(\tau_{\Delta} \geq T_{u}^{b}\right) e^{y D_{u, b}}\right] \\
& =E\left[I\left(\tau_{\triangle}<T_{u}^{b}\right) M_{3}\left(\triangle, y e^{-\alpha \tau_{\Delta}}, b\right)\right]+P\left(\tau_{\triangle} \geq T_{u}^{b}\right) \\
& =M_{3}(\triangle, y, b) E\left[e^{-\alpha \tau_{\Delta}} I\left(\tau_{\triangle}<T_{u}^{b}\right)\right]+P\left(\tau_{\triangle} \geq T_{u}^{b}\right) \\
& \leq M_{3}(\triangle, y, b)+P\left(\tau_{\triangle} \geq T_{u}^{b}\right) .
\end{aligned}
$$


On the other hand, we have

$$
\begin{aligned}
M_{2}(u, y, b) & \geq E\left[I\left(\tau_{\Delta}<T_{u}^{b}, \tau_{\Delta}=t_{1}\right) e^{y D_{u, b}}\right]+E\left[I\left(\tau_{\Delta}<T_{u}^{b}\right) e^{y D_{u, b}}\right] \\
& =E\left[I\left(\tau_{\Delta}<T_{u}^{b}, \tau_{\Delta}=t_{1}\right) M_{3}\left(\triangle, y e^{-\alpha t_{1}}, b\right)\right]+P\left(\tau_{\Delta} \geq T_{u}^{b}\right) \\
& =M_{3}(\triangle, y, b) e^{-\alpha t_{1}} P\left(T_{1}>t_{1}\right)+P\left(\tau_{\Delta} \geq T_{u}^{b}\right) \\
& \geq e^{-(\lambda+\alpha) t_{1}} M_{3}(\triangle, y, b)+P\left(\tau_{\Delta} \geq T_{u}^{b}\right),
\end{aligned}
$$

where $T_{1}$ is the first claim time. As $u \uparrow \triangle, \tau_{\triangle}$ and $t_{1}$ both tend to zero, and $\lim _{u \uparrow \Delta} P\left(\tau_{\triangle} \geq\right.$ $\left.T_{u}^{b}\right)=0$; letting $u \uparrow \triangle$ in (2.11) and (2.12), we get $M_{2}(\triangle-, y, b)=M_{3}(\triangle, y, b)$.

Further, letting $u \uparrow 0$ in (2.1), $u \downarrow 0$ in (2.2), and using (2.6), and then letting $u \uparrow \Delta$ in (2.2), $u \downarrow \Delta$ in (2.3), and using (2.6), we get (2.7).

Remark 2.1 When $\Delta=0$, the conclusions are consistent with Wang et al. [4]. Write

$$
V_{n}(u, y, b)= \begin{cases}V_{n 1}(u, y, b), & -\frac{c}{\beta}<u<0 \\ V_{n 2}(u, y, b), & 0 \leq u<\Delta \\ V_{n 3}(u, y, b), & \Delta \leq u \leq b\end{cases}
$$

Theorem 2.2 The moment of the discounted dividend payments until absolute ruin satisfies the following integro-differential equations:

$$
\begin{aligned}
& (\beta u+c) V_{n 1}^{\prime}(u, b)-(\lambda+n \alpha) V_{n 1}(u, b) \\
& +\lambda \int_{0}^{u+\frac{c}{\beta}} V_{n 1}(u-x, b) d F(x)=0, \quad-\frac{c}{\beta}<u<0, \\
& c V_{n 2}^{\prime}(u, b)-(\lambda+n \alpha) V_{n 2}(u, b)+\lambda \int_{0}^{u} V_{n 2}(u-x, b) d F(x) \\
& \quad+\lambda \int_{u}^{u+\frac{c}{\beta}} V_{n 1}(u-x, b) d F(x)=0
\end{aligned}
$$

for $0 \leq u<\triangle$, and, for $\Delta \leq u \leq b$,

$$
\begin{aligned}
& {[r(u-\triangle)+c] V_{n 3}^{\prime}(u, b)-(\lambda+n \alpha) V_{n 3}(u, b)+\lambda \int_{0}^{u-\Delta} V_{n 3}(u-x, b) d F(x)} \\
& \quad+\lambda \int_{u-\Delta}^{u} V_{n 2}(u-x, b) d F(x)+\lambda \int_{u}^{u+\frac{c}{\beta}} V_{n 1}(u-x, b) d F(x)=0
\end{aligned}
$$

with the following conditions:

$$
\begin{array}{ll}
V_{n 1}\left(-\frac{c}{\beta}, b\right)=0, & \\
\left.V_{n 3}^{\prime}(u, b)\right|_{u=b}=n V_{(n-1) 3}(b, b), \\
V_{n 1}(0-, b)=V_{n 2}(0, b), & V_{n 2}(\triangle-, b)=V_{n 3}(\triangle, b), \\
V_{n 1}^{\prime}(0-, b)=V_{n 2}^{\prime}(0, b), & V_{n 2}^{\prime}(\triangle-, b)=V_{n 3}^{\prime}(\triangle, b) .
\end{array}
$$


Proof The proof is obvious and we omit it here.

Corollary 2.1 For $n=1$, we retain the risk process, and indeed (2.13), (2.14), and (2.15) can be simplified to

$$
\begin{aligned}
& (\beta u+c) V_{11}^{\prime}(u, b)-(\lambda+\alpha) V_{11}(u, b) \\
& +\lambda \int_{0}^{u+\frac{c}{\beta}} V_{11}(u-x, b) d F(x)=0, \quad-\frac{c}{\beta}<u<0, \\
& c V_{12}^{\prime}(u, b)-(\lambda+\alpha) V_{12}(u, b)+\lambda \int_{0}^{u} V_{12}(u-x, b) d F(x) \\
& \quad+\lambda \int_{u}^{u+\frac{c}{\beta}} V_{11}(u-x, b) d F(x)=0
\end{aligned}
$$

for $0 \leq u<\triangle$, and for $\triangle \leq u \leq b$,

$$
\begin{aligned}
& {[r(u-\Delta)+c] V_{13}^{\prime}(u, b)-(\lambda+\alpha) V_{13}(u, b)+\lambda \int_{0}^{u-\Delta} V_{13}(u-x, b) d F(x)} \\
& \quad+\lambda \int_{u-\Delta}^{u} V_{12}(u-x, b) d F(x)+\lambda \int_{u}^{u+\frac{c}{\beta}} V_{11}(u-x, b) d F(x)=0 .
\end{aligned}
$$

Correspondingly, the boundary condition can be simplified to

$$
\begin{array}{ll}
V_{11}\left(-\frac{c}{\beta}, b\right)=0, & \\
\left.V_{13}^{\prime}(u, b)\right|_{u=b}=1, & \\
V_{11}(0-, b)=V_{12}(0, b), & V_{12}(\triangle-, b)=V_{13}(\triangle, b), \\
V_{11}^{\prime}(0-, b)=V_{12}^{\prime}(0, b), & V_{12}^{\prime}(\triangle-, b)=V_{13}^{\prime}(\triangle, b) .
\end{array}
$$

Remark 2.2 When $\Delta=0,(2.20)$ and (2.22) are reduced to (2.1) and (2.2) of Yuen et al. [3], and (2.23)-(2.26) are reduced to $\left(\mathrm{A}_{1}\right)-\left(\mathrm{A}_{4}\right)$ of Yuen et al. [3], respectively.

\section{Explicit expressions for exponential claims}

In this section, we assume that $F(x)=1-e^{-\frac{x}{\mu}}, x>0, \mu>0$, namely, the claim size distribution $F(x)$ is the exponential distribution with mean $\mu$. We obtain explicit expressions of the moment-generating function and higher moments of the discounted dividends.

Substituting $F(x)=1-e^{-\frac{x}{\mu}}$ into (2.13), (2.14), and (2.15), we obtain:

$$
\begin{aligned}
(\beta u+c) V_{n 1}^{\prime}(u, b)= & (\lambda+n \alpha) V_{n 1}(u, b) \\
& -\frac{\lambda}{\mu} e^{-\frac{u}{\mu}} \int_{-\frac{c}{\beta}}^{u} V_{n 1}(x, b) e^{\frac{x}{\mu}} d x, \quad-\frac{c}{\beta}<u<0, \\
c V_{n 2}^{\prime}(u, b)= & (\lambda+n \alpha) V_{n 2}(u, b) \\
& -\frac{\lambda}{\mu} e^{-\frac{u}{\mu}}\left(\int_{-\frac{c}{\beta}}^{0} V_{n 1}(x, b) e^{\frac{x}{\mu}} d x+\int_{0}^{u} V_{n 2}(x, b) e^{\frac{x}{\mu}} d x\right)
\end{aligned}
$$


for $0 \leq u<\Delta$ and, for $\Delta \leq u \leq b$,

$$
\begin{aligned}
{[r(u-\triangle)+c] V_{n 3}^{\prime}(u, b)=} & (\lambda+n \alpha) V_{n 3}(u, b)-\frac{\lambda}{\mu} e^{-\frac{u}{\mu}}\left(\int_{\Delta}^{u} V_{n 3}(x, b) e^{\frac{x}{\mu}} d x\right. \\
& \left.+\int_{0}^{\Delta} V_{n 2}(x, b) e^{\frac{x}{\mu}} d x+\int_{-\frac{c}{\beta}}^{0} V_{n 1}(x, b) e^{\frac{x}{\mu}} d x\right) .
\end{aligned}
$$

Applying the operator $\frac{d}{d u}+\frac{1}{\mu}$ on (3.1), (3.2), and (3.3), respectively, we obtain:

$$
\begin{aligned}
& (\beta u+c) V_{n 1}^{\prime \prime}(u, b)+\left(\frac{\beta u+c}{\mu}+\beta-(\lambda+n \alpha)\right) V_{n 1}^{\prime}(u, b)-\frac{n \alpha}{\mu} V_{n 1}(u, b)=0, \\
& c V_{n 2}^{\prime \prime}(u, b)+\left(\frac{c}{\mu}-(\lambda+n \alpha)\right) V_{n 2}^{\prime}(u, b)-\frac{n \alpha}{\mu} V_{n 2}(u, b)=0, \\
& {[r(u-\triangle)+c] V_{n 3}^{\prime \prime}(u, b)+\left(\frac{r(u-\triangle)+c}{\mu}+r-(\lambda+n \alpha)\right) V_{n 3}^{\prime}(u, b)} \\
& \quad-\frac{n \alpha}{\mu} V_{n 3}(u, b)=0 .
\end{aligned}
$$

Letting $V_{n 1}(u, b)=g_{n}(z)$ and $z=-\frac{\beta u+c}{\beta \mu}$ for $-\frac{c}{\beta}<u<0,(3.4)$ is reduced to

$$
z g_{n}^{\prime \prime}(z)+\left(1-\frac{\lambda+n \alpha}{\beta}-z\right) g_{n}^{\prime}(z)+\frac{n \alpha}{\beta} g_{n}(z)=0
$$

By Slater [14], p.5, the solution of this equation is of the form

$$
\begin{aligned}
g_{n}(z)= & a_{n 1} z^{\frac{\lambda+n \alpha}{\beta}} e^{z} U\left(1+\frac{n \alpha}{\beta}, 1+\frac{\lambda+n \alpha}{\beta},-z\right) \\
& +a_{n 2} z^{\frac{\lambda+n \alpha}{\beta}} e^{z} M\left(1+\frac{n \alpha}{\beta}, 1+\frac{\lambda+n \alpha}{\beta},-z\right),
\end{aligned}
$$

where $a_{n 1}$ and $a_{n 2}$ are arbitrary constants,

$$
\begin{aligned}
& M(a, v, x)=\frac{\Gamma(v)}{\Gamma(v-a) \Gamma(a)} \int_{0}^{1} e^{x t} t^{a-1}(1-t)^{v-a-1} d t, \quad v>a>0, \\
& U(a, v, x)=\frac{1}{\Gamma(a)} \int_{0}^{\infty} e^{-x t} t^{a-1}(1+t)^{v-a-1} d t, \quad a>0,
\end{aligned}
$$

are the confluent hypergeometric functions of the first and second kinds, respectively. Then

$$
V_{n 1}(u, b)=g_{n}\left(-\frac{\beta u+c}{\beta \mu}\right)=a_{n 1} h_{n 1}(u)+a_{n 2} h_{n 2}(u)
$$

with

$$
\begin{aligned}
& h_{n 1}(u)=\left(-\frac{\beta u+c}{\beta \mu}\right)^{\frac{\lambda+n \alpha}{\beta}} e^{-\frac{\beta u+c}{\beta \mu}} U\left(1+\frac{n \alpha}{\beta}, 1+\frac{\lambda+n \alpha}{\beta}, \frac{\beta u+c}{\beta \mu}\right), \\
& h_{n 2}(u)=\left(-\frac{\beta u+c}{\beta \mu}\right)^{\frac{\lambda+n \alpha}{\beta}} e^{-\frac{\beta u+c}{\beta \mu}} M\left(1+\frac{n \alpha}{\beta}, 1+\frac{\lambda+n \alpha}{\beta}, \frac{\beta u+c}{\beta \mu}\right) .
\end{aligned}
$$


We know that

$$
\lim _{u \downarrow-\frac{c}{\beta}} h_{n 1}(u)=\frac{\Gamma\left(\frac{\lambda+n \alpha}{\beta}\right)}{\Gamma\left(\frac{\beta+n \alpha}{\beta}\right)}, \quad \lim _{u \downarrow-\frac{c}{\beta}} h_{n 2}(u)=0 .
$$

Letting $u \downarrow-\frac{c}{\beta}$ on both sides of (3.7) and substituting (2.16) and (3.8) into it, we obtain $a_{n 1}=0$, and thus

$$
V_{n 1}(u, b)=a_{n 2} h_{n 2}(u) .
$$

It follows from (3.5) that $V_{n 2}(u, b)$ takes the form

$$
V_{n 2}(u, b)=a_{n 3} e^{s_{11} u}+a_{n 4} e^{s_{n 2} u},
$$

where $a_{n 3}$ and $a_{n 4}$ are arbitrary constants, and $s_{n 1}, s_{n 2}$ are the two roots of the following equation:

$$
s^{2}+\left(\frac{1}{\mu}-\frac{n \alpha+\lambda}{c}\right) s-\frac{n \alpha}{\mu c}=0
$$

with $p_{n}=\frac{1}{\mu}-\frac{n \alpha+\lambda}{c}, q_{n}=-\frac{n \alpha}{\mu c}$, that is,

$$
s_{n 1}=\frac{-p_{n}+\sqrt{p_{n}^{2}-4 q_{n}}}{2}, \quad s_{n 2}=\frac{-p_{n}-\sqrt{p_{n}^{2}-4 q_{n}}}{2} .
$$

On the other hand, let $V_{n 3}(u, b)=j_{n}(y)$ and $y=-\frac{r u+c-r \Delta}{r \mu}$. Similarly to (3.4), we obtain the solution of (3.6):

$$
\begin{aligned}
j_{n}(y)= & a_{n 5} y^{\frac{\lambda+n \alpha}{r}} e^{y} U\left(1+\frac{n \alpha}{r}, 1+\frac{\lambda+n \alpha}{r},-y\right) \\
& +a_{n 6} y^{\frac{\lambda+n \alpha}{r}} e^{y} M\left(1+\frac{n \alpha}{r}, 1+\frac{\lambda+n \alpha}{r},-y\right) .
\end{aligned}
$$

So we get

$$
V_{n 3}(u, b)=j_{n}\left(-\frac{r u+c-r \Delta}{r \mu}\right)=a_{n 5} h_{n 3}(u)+a_{n 6} h_{n 4}(u),
$$

where

$$
\begin{aligned}
& h_{n 3}(u)=e^{-\frac{r u+c-r \Delta}{r \mu}}\left(-\frac{r u+c-r \Delta}{r \mu}\right)^{\frac{\lambda+n \alpha}{r}} U\left(1+\frac{n \alpha}{r}, 1+\frac{\lambda+n \alpha}{r}, \frac{r u+c-r \Delta}{r \mu}\right), \\
& h_{n 4}(u)=e^{-\frac{r u+c-r \Delta}{r \mu}}\left(-\frac{r u+c-r \Delta}{r \mu}\right)^{\frac{\lambda+n \alpha}{r}} M\left(1+\frac{n \alpha}{r}, 1+\frac{\lambda+n \alpha}{r}, \frac{r u+c-r \Delta}{r \mu}\right),
\end{aligned}
$$

and $a_{n 5}$ and $a_{n 6}$ are arbitrary constants. 
When $n=1$, since $a_{11}=0$, we can get explicit values of $a_{12}, a_{13}, \ldots, a_{16}$. By (2.23)-(2.26) we obtain the following equations:

$$
\left\{\begin{array}{l}
a_{15} h_{13}^{\prime}(b)+a_{16} h_{14}^{\prime}(b)=1, \\
a_{12} h_{12}(0)=a_{13}+a_{14}, \\
a_{13} e^{s_{11} \Delta}+a_{14} e^{s_{12} \Delta}=a_{15} h_{13}(\triangle)+a_{16} h_{14}(\triangle), \\
a_{12} h_{12}^{\prime}(0)=a_{13} s_{11}+a_{14} s_{12}, \\
a_{13} s_{11} e^{s_{11} \triangle}+a_{14} s_{12} e^{s_{12} \Delta}=a_{15} h_{13}^{\prime}(\triangle)+a_{16} h_{14}^{\prime}(\triangle) .
\end{array}\right.
$$

Solving this system, we obtain:

$$
\left\{\begin{array}{l}
a_{12}=\frac{\left[h_{14}(\Delta) h_{13}(\Delta) C_{12} B_{1}-h_{14}^{\prime}(\Delta) h_{13}(\Delta) C_{12} A_{1}+h_{14}^{2}(\Delta) C_{11} B_{1}-h_{14}^{\prime}(\Delta) h_{14}(\Delta) C_{11} A_{1}\right]\left(s_{12}-s_{11}\right)}{\left(h_{12}^{\prime}(0)-s_{11} h_{12}(0)\right) C_{11} C_{12} A_{1}} \\
a_{13}=\frac{\left[h_{14}(\Delta) h_{13}(\Delta) C_{12} B_{1}-h_{14}^{\prime}(\Delta) h_{13}(\Delta) C_{12} A_{1}+h_{14}^{2}(\Delta) C_{11} B_{1}-h_{14}^{\prime}(\Delta) h_{14}(\Delta) C_{11} A_{1}\right]\left(s_{12} h_{12}(0)-h_{12}^{\prime}(0)\right)}{\left(h_{12}^{\prime}(0)-s_{11} h_{12}(0)\right) C_{11} C_{12} A_{1}} \\
a_{14}=\frac{h_{14}(\Delta) h_{13}(\Delta) C_{12} B_{1}-h_{14}^{\prime}(\Delta) h_{13}(\Delta) C_{12} A_{1}+h_{14}^{2}(\Delta) C_{11} B_{1}-h_{14}^{\prime}(\Delta) h_{14}(\Delta) C_{11} A_{1}}{C_{11} C_{12} A_{1}} \\
a_{15}=\frac{h_{14}(\Delta) B_{1}-h_{14}^{\prime}(\Delta) A_{1}}{C_{11}} \\
a_{16}=\frac{h_{13}(\Delta) B_{1}-h_{13}^{\prime}(\Delta) A_{1}}{C_{12}}
\end{array}\right.
$$

where

$$
\begin{aligned}
& A_{1}=\frac{\left(s_{12} h_{12}(0)-h_{12}^{\prime}(0)\right) e^{s_{11} \Delta}+\left(h_{12}^{\prime}(0)-s_{11} h_{12}(0)\right) e^{s_{12} \Delta}}{h_{12}^{\prime}(0)-s_{11} h_{12}(0)}, \\
& B_{1}=\frac{\left(s_{12} h_{12}(0)-h_{12}^{\prime}(0)\right) s_{11} e^{s_{11} \Delta}+\left(h_{12}^{\prime}(0)-s_{11} h_{12}(0)\right) s_{12} e^{s_{12} \Delta}}{h_{12}^{\prime}(0)-s_{11} h_{12}(0)}, \\
& C_{11}=\left[h_{13}^{\prime}(b) h_{14}(\triangle)-h_{13}(\triangle) h_{14}^{\prime}(b)\right] B_{1}+\left[h_{13}^{\prime}(\triangle) h_{14}^{\prime}(b)-h_{13}^{\prime}(b) h_{14}^{\prime}(\triangle)\right] A_{1}, \\
& C_{12}=\left[h_{14}^{\prime}(b) h_{13}(\triangle)-h_{14}(\triangle) h_{13}^{\prime}(b)\right] B_{1}+\left[h_{13}^{\prime}(b) h_{14}^{\prime}(\triangle)-h_{13}^{\prime}(\triangle) h_{14}^{\prime}(b)\right] A_{1} .
\end{aligned}
$$

When $n \geq 2$, by (2.16)-(2.19) we obtain the following equations:

$$
\left\{\begin{array}{l}
a_{n 5} h_{n 3}^{\prime}(b)+a_{n 6} h_{n 4}^{\prime}(b)=n V_{(n-1) 3}^{\prime}(b, b), \\
a_{n 2} h_{n 2}(0)=a_{n 3}+a_{n 4}, \\
a_{n 3} e^{s_{n 1} \Delta}+a_{n 4} e^{s_{n 2} \triangle}=a_{n 5} h_{n 3}(\triangle)+a_{n 6} h_{n 4}(\triangle), \\
a_{n 2} h_{n 2}^{\prime}(0)=a_{n 3} s_{n 1}+a_{n 4} s_{n 2}, \\
a_{n 3} s_{n 1} e^{s_{n 1} \Delta}+a_{n 4} s_{n 2} e^{s_{n 2} \Delta}=a_{n 5} h_{n 3}^{\prime}(\triangle)+a_{n 6} h_{n 4}^{\prime}(\triangle)
\end{array}\right.
$$

Solving this system of equations, we obtain:

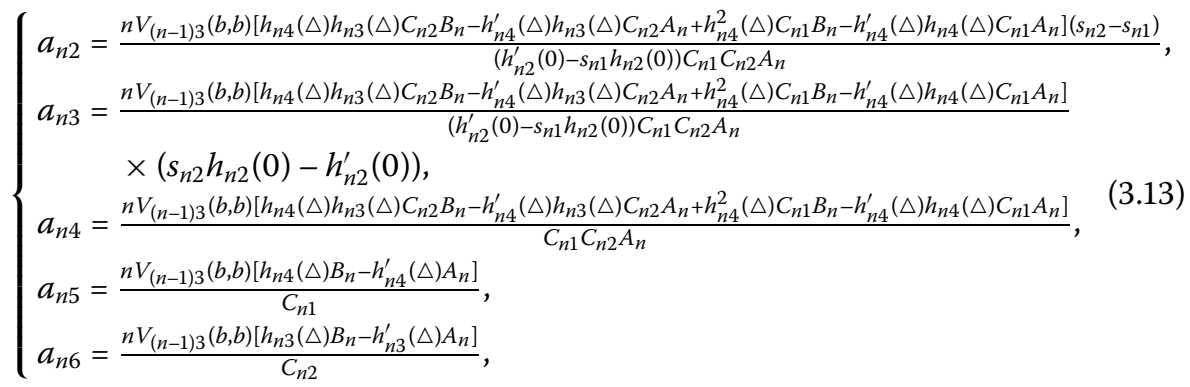


where

$$
\begin{aligned}
& A_{n}=\frac{\left(s_{n 2} h_{n 2}(0)-h_{n 2}^{\prime}(0)\right) e^{s_{n 1} \Delta}+\left(h_{n 2}^{\prime}(0)-s_{n 1} h_{n 2}(0)\right) e^{s_{n 2} \Delta}}{h_{n 2}^{\prime}(0)-s_{n 1} h_{n 2}(0)}, \\
& B_{n}=\frac{\left(s_{n 2} h_{n 2}(0)-h_{n 2}^{\prime}(0)\right) s_{n 1} e^{s_{n 1} \Delta}+\left(h_{n 2}^{\prime}(0)-s_{n 1} h_{n 2}(0)\right) s_{n 2} e^{s_{n 2} \Delta}}{h_{n 2}^{\prime}(0)-s_{n 1} h_{n 2}(0)}, \\
& C_{n 1}=\left[h_{n 3}^{\prime}(b) h_{n 4}(\triangle)-h_{n 3}(\Delta) h_{n 4}^{\prime}(b)\right] B_{n}+\left[h_{n 3}^{\prime}(\Delta) h_{n 4}^{\prime}(b)-h_{n 3}^{\prime}(b) h_{n 4}^{\prime}(\Delta)\right] A_{n}, \\
& C_{n 2}=\left[h_{n 4}^{\prime}(b) h_{n 3}(\triangle)-h_{n 4}(\Delta) h_{n 3}^{\prime}(b)\right] B_{n}+\left[h_{n 3}^{\prime}(b) h_{n 4}^{\prime}(\triangle)-h_{n 3}^{\prime}(\Delta) h_{n 4}^{\prime}(b)\right] A_{n} .
\end{aligned}
$$

From (3.11) and $a_{15}, a_{16}$ in (3.12) we can obtain $V_{13}(b, b)$ see the following Examples 1-4. Recursively, we can obtain explicit expressions of $V_{n 1}(u, b), V_{n 2}(u, b)$, and $V_{n 3}(u, b)$.

We summarize the exact solution for $V_{n 1}(u, b), V_{n 2}(u, b)$, and $V_{n 3}(u, b)$ in the following theorem.

Theorem 3.1 Suppose that the claim size distribution is the exponential distribution with $F(x)=1-e^{-\frac{x}{\mu}}$. Then $V_{n}(u, b)$ is given by

$$
\begin{aligned}
& V_{n 1}(u, b)=a_{n 2} h_{n 2}(u), \quad-\frac{c}{\beta}<u<0, \\
& V_{n 2}(u, b)=a_{n 3} e^{s_{n 1} u}+a_{n 4} e^{s_{n 2} u}, \quad 0 \leq u<\Delta, \\
& V_{n 3}(u, b)=a_{n 5} h_{n 3}(u)+a_{n 6} h_{n 4}(u), \quad \Delta \leq u \leq b .
\end{aligned}
$$

Here, $a_{12}, a_{13}, \ldots, a_{16}$ are obtained in (3.12) for $n=1$; otherwise, for $n \geq 2, a_{n 2}, a_{n 3}, \ldots, a_{n 6}$ are obtained by (3.13).

Remark 3.1 In fact, we can obtain the following explicit expressions of $M_{1}(u, y, b)$, $M_{2}(u, y, b)$, and $M_{3}(u, y, b)$ :

$$
\begin{aligned}
& M_{1}(u, y, b)=1+\sum_{n=1}^{\infty} \frac{y^{n}}{n !} a_{n 2} h_{n 2}(u), \quad-\frac{c}{\beta}<u<0, \\
& M_{2}(u, y, b)=1+\sum_{n=1}^{\infty} \frac{y^{n}}{n !}\left(a_{n 3} e^{s_{n 1} u}+a_{n 4} e^{s_{n 2} u}\right), \quad 0 \leq u<\Delta, \\
& M_{3}(u, y, b)=1+\sum_{n=1}^{\infty} \frac{y^{n}}{n !}\left(a_{n 5} h_{n 3}(u)+a_{n 6} h_{n 4}(u)\right), \quad \Delta \leq u \leq b .
\end{aligned}
$$

At the end of this section, we use the following numerical examples to discuss the impact of the model parameters on the expected dividend payments.

Example 1 Table 1 provides numerical results for $V_{13}(u, b)$ for various $u$ and $r$. We find out that $V_{13}(u, b)$ increases as the credit interest or the initial surplus increases.

Example 2 Table 2 provides numerical results for $V_{13}(u, b)$ for various $u$ and $\beta$. We find out that $V_{13}(u, b)$ decreases as the force of debit interest increases but increases as the initial surplus increases. 
Table 1 Influence of $u$ and $r$ on $V_{13}(u, b)$ with $b=2.8, \Delta=1.5, \alpha=0.03, \mu=1, \lambda=1, c=1.5$, $\beta=0.09$

\begin{tabular}{lllllll}
\hline $\boldsymbol{u} \backslash \boldsymbol{r}$ & $\mathbf{0 . 0 3}$ & $\mathbf{0 . 0 4}$ & $\mathbf{0 . 0 5}$ & $\mathbf{0 . 0 6}$ & $\mathbf{0 . 0 7}$ & $\mathbf{0 . 0 8}$ \\
\hline 1.6 & 27.8537 & 28.1717 & 28.4903 & 28.8095 & 29.1294 & 29.4498 \\
1.7 & 27.9233 & 28.2420 & 28.5614 & 28.8813 & 29.2018 & 29.5230 \\
1.8 & 27.9961 & 28.3155 & 28.6355 & 28.9562 & 29.2744 & 29.5992 \\
1.9 & 28.0719 & 28.3920 & 28.7127 & 29.0340 & 29.3559 & 29.6784 \\
2.0 & 28.1507 & 28.4714 & 28.7928 & 29.1147 & 29.4372 & 29.7603 \\
2.1 & 28.2323 & 28.5537 & 28.8756 & 29.1981 & 29.5212 & 29.8449 \\
2.2 & 28.3167 & 28.6386 & 28.9610 & 29.2840 & 29.6077 & 29.9318 \\
2.3 & 28.4038 & 28.7261 & 29.0490 & 29.3725 & 29.6965 & 30.0212 \\
2.4 & 28.4933 & 28.8161 & 29.1394 & 29.4632 & 29.7877 & 30.1127 \\
\hline
\end{tabular}

Table 2 Influence of $u$ and $\beta$ on $V_{13}(u, b)$ with $b=2.8, \Delta=1.5, \alpha=0.03, \mu=1, \lambda=1, c=1.5$, $r=0.04$

\begin{tabular}{lllllll}
\hline $\boldsymbol{u} \backslash \boldsymbol{\beta}$ & $\mathbf{0 . 0 9}$ & $\mathbf{0 . 1 0}$ & $\mathbf{0 . 1 1}$ & $\mathbf{0 . 1 2}$ & $\mathbf{0 . 1 3}$ & $\mathbf{0 . 1 4}$ \\
\hline 1.6 & 28.1717 & 27.1050 & 26.1146 & 25.1984 & 24.3525 & 23.5719 \\
1.7 & 28.2420 & 27.1783 & 26.1906 & 25.2769 & 24.4333 & 23.6549 \\
1.8 & 28.3155 & 27.2544 & 26.2691 & 25.3577 & 24.5162 & 23.7396 \\
1.9 & 28.3920 & 27.3332 & 26.3501 & 25.4407 & 24.6010 & 23.8262 \\
2.0 & 28.4714 & 27.4147 & 26.4334 & 25.5258 & 24.6877 & 23.9144 \\
2.1 & 28.5537 & 27.4987 & 26.5191 & 25.6129 & 24.7763 & 24.0042 \\
2.2 & 28.6386 & 27.5851 & 26.6069 & 25.7020 & 24.8666 & 24.0956 \\
2.3 & 28.7261 & 27.6738 & 29.6968 & 25.7930 & 24.9585 & 24.1884 \\
2.4 & 28.8161 & 27.7648 & 26.7887 & 25.8857 & 25.0521 & 24.2827 \\
\hline
\end{tabular}

Table 3 Influence of $u$ and $\Delta$ on $V_{13}(u, b)$ with $b=2.8, \alpha=0.03, \mu=1, \lambda=1, c=1.5, \beta=0.09$, $r=0.04$

\begin{tabular}{lllllll}
\hline $\boldsymbol{u} \backslash \boldsymbol{\Delta}$ & $\mathbf{0 . 9}$ & $\mathbf{1 . 1}$ & $\mathbf{1 . 3}$ & $\mathbf{1 . 5}$ & $\mathbf{1 . 7}$ & $\mathbf{1 . 9}$ \\
\hline 1.6 & 20.8956 & 22.6238 & 24.9465 & 28.1717 & 32.8679 & 40.2129 \\
1.7 & 20.9881 & 22.7109 & 25.0265 & 28.2420 & 32.9245 & 40.2483 \\
1.8 & 21.0815 & 22.7994 & 25.1086 & 28.3155 & 32.9857 & 40.2906 \\
1.9 & 21.1756 & 22.8892 & 25.1928 & 28.3920 & 33.0512 & 40.3394 \\
2.0 & 21.2706 & 22.9803 & 25.2789 & 28.4714 & 33.1211 & 40.3944 \\
2.1 & 21.3662 & 23.0727 & 25.3669 & 28.5537 & 33.1950 & 40.4556 \\
2.2 & 21.4625 & 23.1662 & 25.4568 & 28.6386 & 33.2779 & 40.5256 \\
2.3 & 21.5594 & 23.2608 & 25.5484 & 28.7261 & 33.3545 & 40.5952 \\
2.4 & 21.6570 & 23.3565 & 25.6417 & 28.8161 & 33.4398 & 40.6732 \\
\hline
\end{tabular}

Table 4 Influence of $u$ and $b$ on $V_{13}(u, b)$ with $\Delta=1.5, \alpha=0.03, \mu=1, \lambda=1, c=1.5, \beta=0.09$, $r=0.04$

\begin{tabular}{llllllll}
\hline $\boldsymbol{u} \backslash \boldsymbol{b}$ & $\mathbf{2 . 5}$ & $\mathbf{2 . 6}$ & $\mathbf{2 . 7}$ & $\mathbf{2 . 8}$ & $\mathbf{2 . 9}$ & $\mathbf{3 . 0}$ & $\mathbf{3 . 1}$ \\
\hline 1.6 & 30.1186 & 29.4128 & 28.7660 & 28.1717 & 27.6244 & 27.1195 & 26.6526 \\
1.7 & 30.1938 & 29.4862 & 28.8378 & 28.2420 & 27.6934 & 27.1872 & 26.7192 \\
1.8 & 30.2724 & 29.5630 & 28.9128 & 28.3155 & 27.7655 & 27.2579 & 26.7887 \\
1.9 & 30.3542 & 29.6428 & 28.9909 & 28.3920 & 27.8405 & 27.3316 & 26.8611 \\
2.0 & 30.4391 & 29.7258 & 29.0720 & 28.4714 & 27.9184 & 27.4080 & 26.9362 \\
2.1 & 30.5270 & 29.8116 & 29.1560 & 28.5537 & 27.9990 & 27.4872 & 27.0140 \\
2.2 & 30.6178 & 29.9003 & 29.2427 & 28.6386 & 28.0823 & 27.5689 & 27.0943 \\
2.3 & 30.7113 & 29.9916 & 29.3321 & 28.7261 & 28.1681 & 27.6532 & 27.1771 \\
2.4 & 30.8075 & 30.0856 & 29.4239 & 28.8161 & 28.2563 & 27.7398 & 27.2623 \\
\hline
\end{tabular}


Example 3 Table 3 provides numerical results for $V_{13}(u, b)$ for various $u$ and $\triangle$. We find out that $V_{13}(u, b)$ increases as the liquid reserve or the initial surplus increases.

Example 4 Table 4 provides numerical results for $V_{13}(u, b)$ for various $u$ and $b$. We find out that $V_{13}(u, b)$ decreases as $b$ increases but increases as the initial surplus increases.

We plot four figures (Figures 1-4) for the surfaces of the expected dividend payments with the help of Tables 1-4, from which we can see the influence of credit interest, debit interest, dividend barrier $b$, and liquid reserve $\Delta$ on the values of the expected dividend payments.

For $\Delta=0$, our model is reduced to Wang and Yin [15]. For example, Figures 5 and 6 show that the expected dividend payments $V(u, b)$ decrease as the debit interest increases but increase as credit interest increases, which is also obtained by Wang and Yin [15], Tables 1 and 2 .
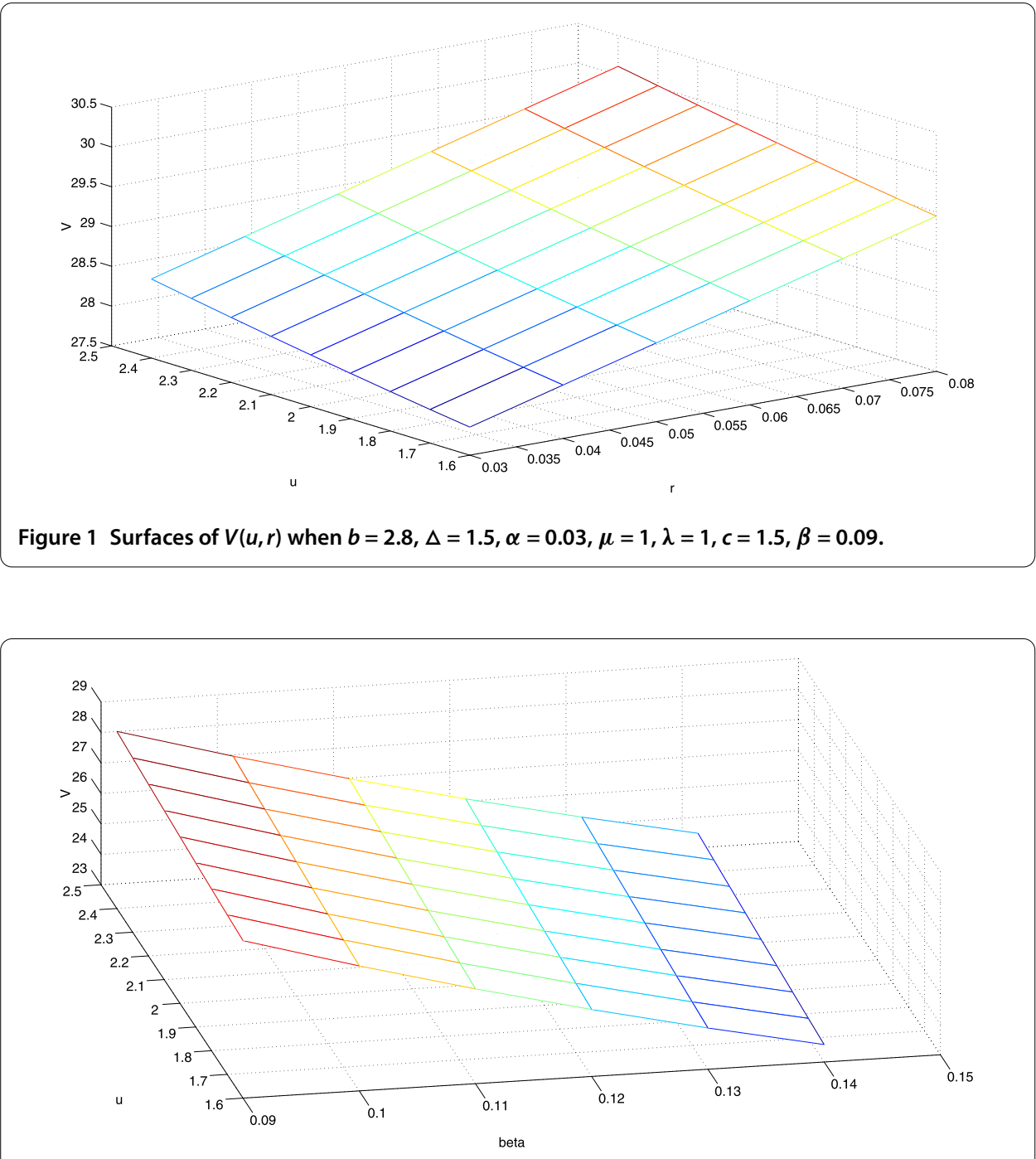

Figure 2 Surfaces of $V(u, \beta)$ when $b=2.8, \Delta=1.5, \alpha=0.03, \mu=1, \lambda=1, c=1.5, r=0.04$. 

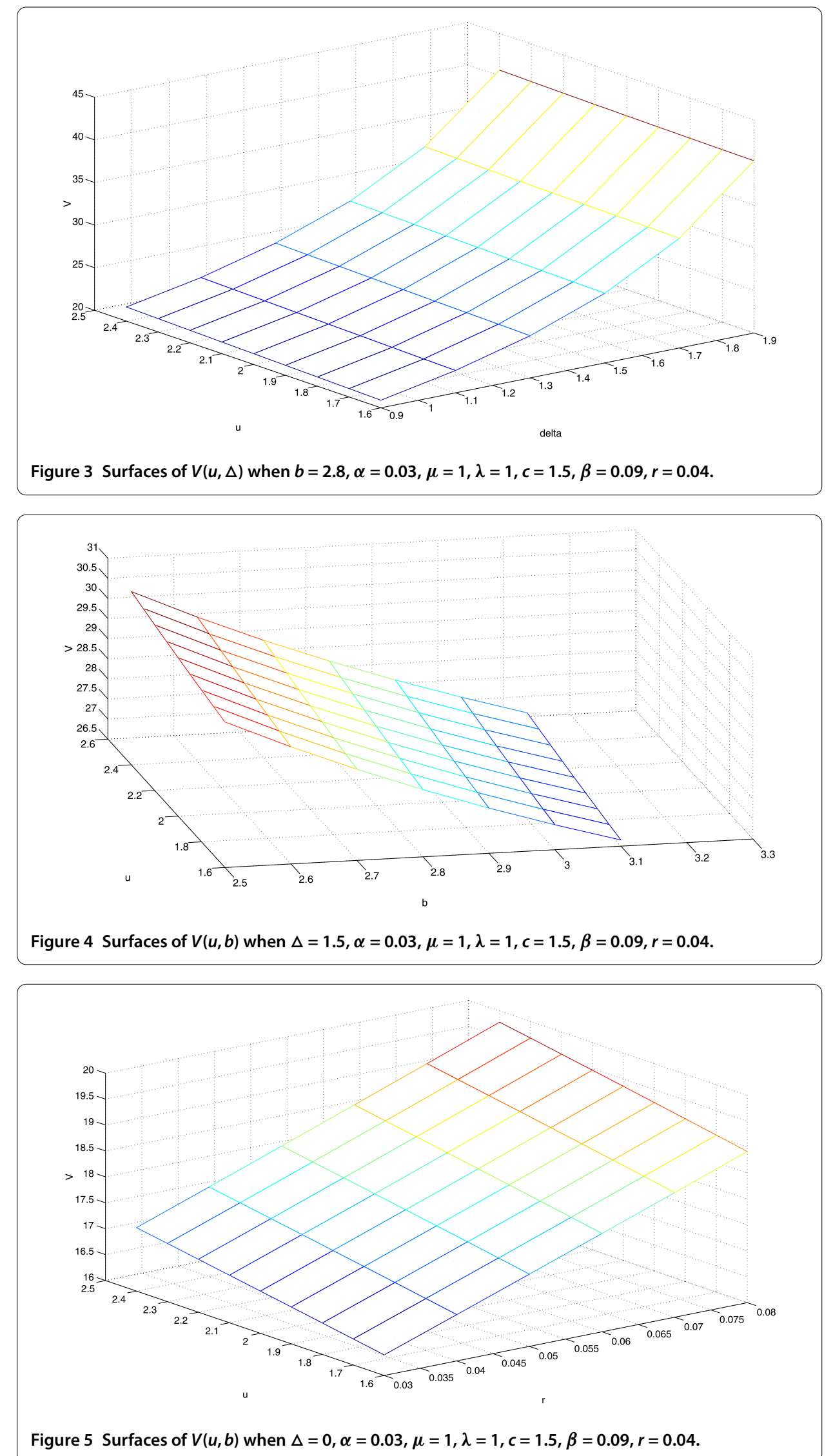


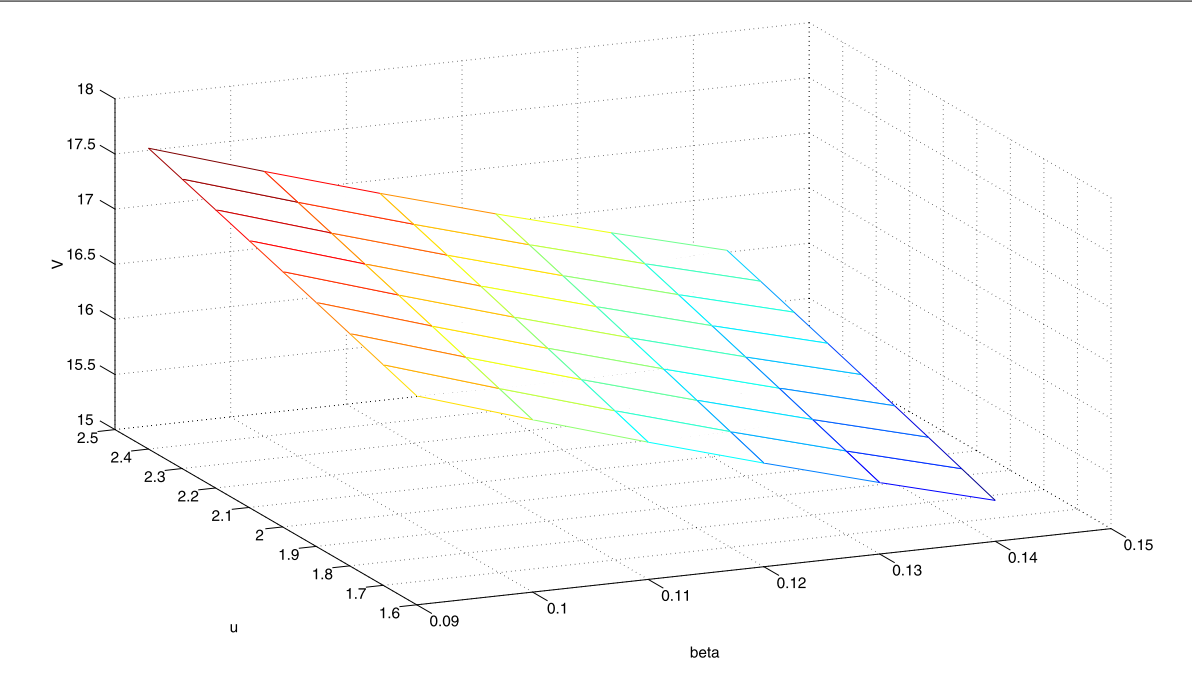

Figure 6 Surfaces of $V(u, b)$ when $\Delta=0, \alpha=0.03, \mu=1, \lambda=1, c=1.5, \beta=0.09, r=0.04$

\section{Competing interests}

The authors declare that they have no competing interests.

\section{Authors' contributions}

DL conceived of the study and drafted the manuscript, DP participated in the design of the study and made the contribution to simulations. ZH participated in its design and helped to draft the manuscript. All authors have read and approved the final manuscript.

\section{Author details}

'School of Mathematics and Computational Science, Hunan University of Science and Technology, Xiangtan, Hunan 411201, P.R. China. ${ }^{2}$ School of Mathematics and Computational Science, Central South University, Changsha, Hunan 410075, P.R. China.

\section{Acknowledgements}

This research is fully supported by a grant from National Natural Science foundation of Hunan (2015JJ6041), by National Natural Science Foundation of China (1 1501191), by National Natural Science Foundation of China, Tian Yuan Foundation (11426100), by National Social Science Fund (15BTJ028), and by National Social Science Fund (13BGL106).

Received: 2 July 2015 Accepted: 10 January 2016 Published online: 10 March 2016

\section{References}

1. Embrechts, P, Schmidli, H: Ruin estimation for a general insurance risk model. Adv. Appl. Probab. 26(2), 404-422 (1994)

2. Cai, J: On the time value of absolute ruin with debit interest. Adv. Appl. Probab. 39(2), 343-359 (2007)

3. Yuen, KC, Zhou, M, Guo, J: On a risk model with debit interest and dividend payments. Stat. Probab. Lett. 78(15), 2426-2432 (2008)

4. Wang, C, Yin, C, Li, E: On the classical risk model with credit and debit interests under absolute ruin. Stat. Probab. Lett. 80(5-6), 427-436 (2010)

5. Yin, C, Wang, C: The perturbed compound Poisson risk process with investment and debit interest. Methodol. Comput. Appl. Probab. 12(3), 391-413 (2010)

6. Cai, J, Feng, R, Willmot, GE: The compound Poisson surplus model with interest and liquid reserves: analysis of the Gerber-Shiu discounted penalty function. Methodol. Comput. Appl. Probab. 11(3), 401-423 (2009)

7. Gao, S, Liu, Z: The perturbed compound Poisson risk model with constant interest and a threshold dividend strategy. J. Comput. Appl. Math. 233(9), 2181-2188 (2010)

8. Gerber, HU, Shiu, ESW: On optimal dividend strategies in the compound Poisson model. N. Am. Actuar. J. 10(2), 76-93 (2006)

9. Lin, XS, Willmot, GE, Drekic, S: The classical risk model with a constant dividend barrier: analysis of the Gerber-Shiu discounted penalty function. Insur. Math. Econ. 33(3), 551-566 (2003)

10. de Finetti, B: Su un'impostazione alternativa dell teoria colletiva del rischio. In: Transactions of the XV International Congress of Actuaries, vol. 2, pp. 433-443 (1957)

11. Albrecher, $H$, Claramunt, MM, Mármol, M: On the distribution of dividend payments in a Sparre Andersen model with generalized Erlang(n) interclaim times. Insur. Math. Econ. 37(2), 324-334 (2005)

12. Cai, J, Feng, R, Willmot, GE: Analysis of the compound Poisson surplus model with liquid reserves, interest and dividends. ASTIN Bull. 39(1), 225-247 (2009)

13. Sendova, KP, Zang, Y: Interest-bearing surplus model with liquid reserves. J. Insur. Issues 33(2), 178-196 (2010)

14. Slater, LJ: Confluent Hypergeometric Functions. Cambridge University Press, London (1960)

15. Wang, C, Yin, C: Dividend payments in the classical risk model under absolute ruin with debit interest. Appl. Stoch. Models Bus. Ind. 25(3), 247-262 (2009) 